Wright State University

CORE Scholar

Obstetrics and Gynecology Faculty Publications

Obstetrics and Gynecology

$2-2012$

\title{
Reducing Emergency Births by Modification in Oxytocin Utilization
}

Gary Ventolini

Michael L. Galloway

Wright State University, michael.galloway@wright.edu

Sheela M. Barhan

Wright State University, sheela.barhan@wright.edu

Marc R. Belcastro

marc.belcastro@wright.edu

Follow this and additional works at: https://corescholar.libraries.wright.edu/obgyn

Part of the Obstetrics and Gynecology Commons

\section{Repository Citation}

Ventolini, G., Galloway, M. L., Barhan, S. M., \& Belcastro, M. R. (2012). Reducing Emergency Births by Modification in Oxytocin Utilization. Journal of Clinical Gyneclology and Obstetrics, 1 (1), 1-3.

https://corescholar.libraries.wright.edu/obgyn/60

This Article is brought to you for free and open access by the Obstetrics and Gynecology at CORE Scholar. It has been accepted for inclusion in Obstetrics and Gynecology Faculty Publications by an authorized administrator of CORE Scholar. For more information, please contact library-corescholar@wright.edu. 


\title{
Reducing Emergency Births by Modification in Oxytocin Utilization
}

\author{
Gary Ventolini ${ }^{\mathrm{a}, \mathrm{c}}$, Michael L. Galloway ${ }^{\mathrm{a}}$, Sheela Barhan ${ }^{\mathrm{a}}$, \\ Marc R. Belcastro ${ }^{b}$
}

\begin{abstract}
Background: The purpose of our study was to explore the correlation between the amount of oxytocin use and emergency vacuum, forceps, cesarean births and neonatal intensive care unit (NICU) response to fetal distress. In 2004, we restricted the criteria for oxytocin utilization for labor induction and augmentation.
\end{abstract}

Methods: This retrospective study was carried out at a large private university tertiary care hospital- affiliated, and included data from the years 2005 to 2007. We utilized hospital data from vital statistics, labor and delivery, central pharmacy and NICU. Information obtained included maternal characteristics, annual birth data, indication and numbers of emergency vacuum, forceps and cesarean births, oxytocin utilization, and number of NICU responses to fetal distress.

Results: The total number of deliveries during the studied period equaled14,184. The oxytocin utilization showed a reduction from $93.3 \%$ to $78.9 \%$. The number of patients who did not receive oxytocin during labor increased from $6.7 \%$ to $21.1 \%$. The correlation between the reduction of oxytocin utilization with the reduction of emergency cesarean births ( $10.9 \%$ to $5.07 \%)$, vacuum briths $(9.1 \%$ to $8.5 \%$ ), and forceps birth (4\% to $2.3 \%$ ) including NICU responses

Manuscript accepted for publication October 31, 2011

${ }^{a}$ Wright State University Boonshoft School of Medicine, Obstetrics and Gynecology Department, 128 East Apple Street, CHE Building, Suite 3800, Dayton, Ohio 45409, USA

${ }^{b}$ Wright State University Boonshoft School of Medicine, 128 East Apple

Street, CHE Building, Suite 3822, Dayton, Ohio 45409, USA

${ }^{\mathrm{c} C o r r e s p o n d i n g ~ a u t h o r: ~ G a r y ~ V e n t o l i n i . ~}$

Email: gary.ventolini@wright.edu

doi:10.4021/jcgo5e to fetal distress $(\mathrm{P}=0.0001)$ revealed a significant statistical difference. The overall cesarean section rate did not indicate a significant increase $29.4 \%$ to $29.8 \%(\mathrm{P}=0.14)$ nor did the patient's characteristics differ.

Conclusions: In our population, reducing oxytocin appears to strongly correlate with a reduction in the number of emergency vacuum, forceps, cesarean births and NICU responses to fetal distress.

Keywords: Emergency; Reducing; Utilization

\section{Introduction}

A national consensus reveals that annual cesarean birth rates in North America are excessive and are increasing [1]. Despite the morbidity associated with cesarean birth, a safe preventive strategy to reduce the use of this delivery modality has not been clearly identified [1]. Morbidity increases dramatically when an instrument is utilized in urgent or emergent situations resulting in a cesarean delivery. Recent data has shown that a standardized regimen of oxytocin has the potential to improve patient safety [1].

The purpose of our study was to explore the correlation between the prevalence of oxytocin use and the utilization rate of emergency vacuum, forceps, cesarean births and neonatal resuscitation team response to fetal distress. There was a national interest in 2004 aimed at reducing the number of cesarean births and a local interest aimed at reducing the utilization of the emergency neonatal resuscitation team responses and the utilization of emergency cesarean, vacuum and forceps in response to fetal distress.

\section{Material and Methods}

The study took place at a large private university tertiary care hospital-affiliated. We analyzed annual data obtained from vital statistics, labor and delivery, central pharmacy and neonatal intensive care units from the period January 2002 to December 2007. Information obtained included maternal 
characteristics, annual birth data, indications and numbers of emergency vacuum, forceps, and cesarean births, oxytocin utilization and number of responses to fetal distress. Additionally, cord prolapse, IUGR, preeclampsia and abruption data was collected. The data was evaluated comparing two groups: 1) historical data from January 2002 to December 2004 and 2) study period data from January 2005 to December 2007.

The pivotal change occurred in January 2005 and involved changing the oxytocin administration protocol for labor induction and labor augmentation as follows: a. initial dose 2 miliunits increasing by 2 every 20 minutes; b. initial dose 2 miliunits increasing by 2 every 30 to 40 minutes.

The protocol was approved by the hospital's OB/GYN department and implemented into the nursing curriculum to be taught to nursing personnel as required by all the obstetrical care providers. Only the patients who received oxytocin for labor induction or augmentation were included in the analysis.

Statistical analysis was performed using GraphPad Software (GraphPad Software Inc., San Diego, California). We used two tailed t-tests for continuous data and chi-square analysis for proportions. A P value of less than 0.05 was considered significant. The Institutional Review Board approved the study.

\section{Results}

The total number of singleton births during the studied period was 14,184 neonates. The patient's characteristics did not differ during the studied period or when compared to the historical data. Birth data was compared by calendar year. The oxytocin utilization that was stable during the historical period, showed a reduction during the study period from $93.3 \%$ to $78.9 \%$ on or after year 1 to year $3(\mathrm{P}=0.0001)$. The amount of oxytocin used by each patient during labor induction was reduced from 51.6 units to 34.8 units $(\mathrm{P}=0.001)$ and from 36.7 units to 22.1 units by each patient during labor augmentation. There was a significant reduction in the number of emergency cesarean births: from $10.9 \%$ to $5.7 \%$ ( $\mathrm{P}=$ $0.0001)$, vacuum births: from $9.1 \%$ to $8.5 \%(\mathrm{P}=0.001)$ and forceps births: from $4 \%$ to $2.3 \%(\mathrm{P}=0.0001)$. The NICU team's responses to fetal distress were also significantly reduced $(\mathrm{P}=0.0001)$.

During the studied period or when compared to the historical data the overall cesarean section rate did not significantly change $(29.4 \%$ to $29.8 \%)(P=0.14)$ nor the number of cord prolapse, IUGR, preeclampsia, and abruption cases.

\section{Discussion}

The change in oxytocin protocol reflects a more natural, physiologic and tolerant perspective for the normal processes of labor. The increasing utilization of the emergency neonatal resuscitation team responses and the utilization of emergency vacuum and forceps in response to fetal distress at our hospital were a major concern. We designed the study to address the issues of emergency births.

In our patient population, the reduction of oxytocin utilization during the labor period after a standardized regimen of oxytocin was implemented and strongly correlates with a reduction on the number of emergency instrumental births including vacuum and forceps assisted and cesarean sections. The NICU team's calls and responses to acute fetal distress also appear to be strongly correlated with the utilization of a low oxytocin protocol. According to the most experienced nursing personnel in our unit, this proved to be not only a less aggressive protocol but the safest protocol.

Limitations of our study included retrospective design and registered data. We considered there could be other contributing factors regarding fewer patients receiving oxytocin. Strengths to be considered included: the data provenance from one large tertiary care hospital, the large number of patients, births, procedures and records analyzed from vital statistics.

Appropriate management of labor and precise interpretation of fetal heart tracing have shown it may also be effective in reducing the cesarean rate. According to Mesleh RA et al [2] patients with one previous cesarean section were 3 times more likely to have a cesarean section as compared to mothers with unscarred uterus.

Reducing the overall cesarean rate is possible by further exploring the primary indication for the first cesarean section. Protocols were used to allow more cases with one or more previous vaginal births after a cesarean section (VBAC) to have a trial of vaginal birth under close monitoring. The exponential increase of malpractice law suits has discouraged this practice with the exception of a few selected hospitals.

Dabbas et al [3] examined 11,506 women who delivered at King Hussein Medical Center for the indications and rate of cesarean section in referral hospitals. He found that some indications for cesarean section such as dystocia and fetal distress were over-utilized resulting in a high CS rate. His research suggests that proper management of labor and precise interpretation of fetal heart tracing might be effective in reducing the cesarean section rate.

Other preventative approaches used to reducing cesarean births have also been reported. A study done by Nicholson et al [4] found that practitioners who often used risk-guided, prostaglandin-assisted labor induction had a lower cesarean delivery rate without increases in rates of other adverse birth outcomes. No randomized controlled trial of this method of care has been reported.

In addition to reducing clinical variation and improving clinical quality of care, Ransom et al [5] suggests that adherence to clinical pathways might protect clinicians and insti- 
tutions against malpractice litigation. A retrospective study was conducted at a large health system delivering 12,000 infants a year was completed to determine whether guideline compliance would affect medico legal risk in obstetrics. The study concluded that litigation could possibly be prevented by closely following clinical pathways, reducing clinical variation, as well as improving the quality of care for the patient.

A meta-analysis completed by Chaillet et al [6] suggests that cesarean section rates can be safely reduced by implementing a plan of care that involves health workers in analyzing and modifying their practice. The study demonstrated that more developed strategies, based on audit and detailed feedback, are designed to improve clinical practice and effectively reduce cesarean section rates.

Gagnon et al [7] conducted a secondary analysis of a randomized controlled trial that compared the advantages of one-to-one labor support to the usual intra partum care that is given to women that are given oxytocin during labor. The analysis concluded that patients stimulated with oxytocin and had continuous one-to-one nursing support were less likely to require a cesarean section.

While our emergency cesarean rate was significantly reduced, our overall cesarean section rate did not significantly change in our population during the studied period compared to the historical data. We did not observe significant change in the number of cord prolapse, IUGR, preeclampsia, and abruption cases. We have to attribute the reduction in oxytocin in our population as a likely contributor to the reduction in the number of emergency instrumental births including vacuum, forceps assisted and cesarean sections.

\section{Conclusion}

In our patient population, reducing oxytocin appears to be a likely contributor to the reduction in the number of emergen- cy instrumental deliveries (vacuum and forceps), cesarean births and NICU team responses to fetal distress.

\section{Acknowledgement}

This abstract was accepted and presented at the ACOG annual clinical meeting in 2009.

\section{References}

1. American College of Obstetricians and Gynecologists ACOG. Induction of Labor. Practice Bulletin \#107, August 2009.

2. Mesleh RA, Kurdi AM, Ayoub HS. Can the rate of cesarean section be reduced? Saudi Med J. 2000;21(11):10541058 .

3. Dabbas M, Al-Sumadi A. Cesarean section rate: much room for reduction. Clin Exp Obstet Gynecol. 2007;34(3):146-148.

4. Nicholson JM, Yeager DL, Macones G. A preventive approach to obstetric care in a rural hospital: association between higher rates of preventive labor induction and lower rates of cesarean delivery. Ann Fam Med. 2007;5(4):310-319.

5. Ransom SB, Studdert DM, Dombowski MP, Mello MM, Brennan TA. Reduced medicolegal risk by compliance with obstetric clinical pathways: a case -control study. Am J Manag Care. 2002 Aug; 8(8): 730-40.

6. Chaillet N, Dumont A. Evidence-based strategies for reducing cesarean section rates: a meta-analysis. Birth. 2007;34(1):53-64.

7. Gagnon AJ, Waghorn K. One-to-one nurse labor support of nulliparous women stimulated with oxytocin. J Obstet Gynecol Neonatal Nurs. 1999;28(4):371-376. 